Open Access

\title{
How to Improve Diagnostic Accuracy in Suspicious Ampullary Lesions?
}

\author{
Woo Hyun Paik ${ }^{1}$ and Jin-Hyeok Hwang ${ }^{2}$ \\ ${ }^{1}$ Department of Internal Medicine, Inje University Ilsan Paik Hospital, Inje University College of Medicine, Goyang, ${ }^{2}$ Department of Internal Medicine, \\ Seoul National University Bundang Hospital, Seoul National University College of Medicine, Seongnam, Korea
}

See "Diagnostic Accuracy of the Initial Endoscopy for Ampullary Tumors" by Hee Seung Lee, Jong Soon Jang, Seungho Lee, et al., on page 239-246.

In cases of ampullary lesions, differential diagnosis between tumors and inflammation by endoscopic findings is sometimes challenging. Even if operators have experience in endoscopic techniques, diagnostic accuracy by gross morphology is only $67 \%$. Although histologic evaluation of ampullary lesions with forceps biopsy is performed during endoscopy, there is still a risk of false negativity for tumorous lesions. It is well known that one or two forceps biopsies are not sufficient for diagnosis during upper or lower endoscopy, and the diagnostic accuracy of the first forceps biopsy was as low as $67 \%$ in this study. Although the results did not reach statistical significance, there was a trend toward improved diagnostic accuracy with more biopsies ( $56 \%$ by two biopsies vs. $75 \%$ by three biopsies). Therefore, multiple ampullary biopsies should be considered as a means of overcoming this problem. However, acute pancreatitis can be a major concern of multiple forceps biopsies. Less invasive and more accurate diagnostic modalities are necessary for the detection and differential diagnosis of ampullary lesions. There have been many developments in diagnostic endoscopy, including endoscopic ultrasound (EUS), intraductal ultrasonography (IDUS), narrow band imaging (NBI), and confocal laser endomicroscopy (CLE). EUS is useful for the detection and differential diagnosis of ampullary tumors, and can also provide more information about the depth of tumor invasion. ${ }^{1}$ IDUS is likely to be superior to EUS in terms of $\mathrm{T}$ staging, but sometimes tends to overestimate tumor Received: April 27, 2015 Accepted: May 7, 2015

Correspondence: Jin-Hyeok Hwang

Department of Internal Medicine, Seoul National University Bundang Hospital, Seoul National University College of Medicine, 82 Gumi-ro 173beon-gil, Bundang-gu, Seongnam 463-707, Korea

Tel: +82-31-787-7009, Fax: +82-31-787-4051, E-mail: woltoong@snu.ac.kr

(@) This is an Open Access article distributed under the terms of the Creative Commons Attribution Non-Commercial License (http://creativecommons.org/ licenses/by-nc/3.0) which permits unrestricted non-commercial use, distribution, and reproduction in any medium, provided the original work is properly cited. invasion into the bile duct., ${ }^{2,3} \mathrm{~A}$ recent retrospective study has reported that NBI was useful for the differential diagnosis of small ampullary lesions. ${ }^{4}$ The usefulness of CLE for the diagnosis of ampullary lesions has not been demonstrated, and is still under investigation since there has been no standardization of CLE image criteria for ampullary tumors. ${ }^{5}$

Although the accuracy of endoscopic imaging and forceps biopsy each was low, the diagnostic accuracy was $91 \%$ when endoscopic diagnoses and biopsy results were concordant. Therefore, the authors conclude that if there is discordance between endoscopic findings and biopsy results, re-evaluation with side-viewing endoscopy after resolution of inflammation is recommended. ${ }^{6}$

In summary, the diagnostic accuracy of first endoscopy for ampullary tumors is reliable if both endoscopic findings and biopsy results are concordant. When both results are repeatedly in disagreement, further evaluation with other diagnostic modalities including EUS, IDUS, and NBI should be considered.

\section{Conflicts of Interest}

The authors have no financial conflicts of interest.

\section{REFERENCES}

1. Artifon EL, Couto D Jr, Sakai P, da Silveira EB. Prospective evaluation of EUS versus CT scan for staging of ampullary cancer. Gastrointest Endosc 2009;70:290-296.

2. Ito K, Fujita N, Noda Y, et al. Preoperative evaluation of ampullary neoplasm with EUS and transpapillary intraductal US: a prospective and histopathologically controlled study. Gastrointest Endosc 2007;66: 740-747.

3. Ito K, Fujita N, Noda Y. Endoscopic diagnosis and treatment of ampullary neoplasm (with video). Dig Endosc 2011;23:113-117.

4. Park JS, Seo DW, Song TJ, et al. Usefulness of white-light imagingguided narrow-band imaging for the differential diagnosis of small ampullary lesions. Gastrointest Endosc 2015 Mar 12 [Epub]. http://dx.doi. 
org/10.1016/j.gie.2014.11.044.

5. Bakhru MR, Sethi A, Jamidar PA, et al. Interobserver agreement for confocal imaging of ampullary lesions: a multicenter single-blinded study. J Clin Gastroenterol 2013;47:440-442.

6. Lee HS, Jang JS, Lee $S$, et al. Diagnostic accuracy of the initial endoscopy for ampullary tumors. Clin Endosc 2015;48:239-246. 